\title{
Comparison of in-house SARS-CoV-2 genome extraction procedures. A need for COVID-19 pandemic
}

Gabriel Martin ( $\square$ gabrielmartinrguez1994@gmail.com )

Hospital Universitario Central de Asturias https://orcid.org/0000-0003-0304-6250

\section{Susana Rojo-Alba}

Hospital Universitario Central de Asturias

Cristian Castelló-Abietar

Hospital Universitario Central de Asturias

Fátima Abreu-Salinas

Hospital Universitario Central de Asturias

Isabel Costales

Hospital Universitario Central de Asturias

Jose Antonio Boga

Hospital Universitario Central de Asturias

Santiago Melón

Hospital Universitario Central de Asturias

Marta Elena Álvarez-Argüelles

Hospital Universitario Central de Asturias

\section{Research Article}

Keywords: SARS-CoV-2, COVID-19, genome extraction, In-house protocol, Heat extraction

Posted Date: March 15th, 2021

DOl: https://doi.org/10.21203/rs.3.rs-214704/v1

License: (c) (i) This work is licensed under a Creative Commons Attribution 4.0 International License. Read Full License

Version of Record: A version of this preprint was published at Journal of Virological Methods on December 1st, 2021. See the published version at https://doi.org/10.1016/j.jviromet.2021.114415. 


\section{Comparison of in-house SARS-CoV-2 genome extraction procedures. A need for COVID-19 pandemic}

Gabriel Martín, Susana Rojo-Alba, Cristian Castelló-Abietar, Fátima AbreuSalinas, Isabel Costales, Jose Antonio Boga, Santiago Melón, Marta Elena ÁlvarezArgüelles.

Servicio de Microbiología. Hospital Universitario Central de Asturias (HUCA) and Grupo de Investigación Microbiología Traslacional, Instituto de Investigación Sanitaria del Principado de Asturias (ISPA), Oviedo. Spain.

Word count: 1757 words

Correspondence:

Servicio de Microbiología.

Hospital Universitario Central de Asturias

Avenida de Roma s / n 33011 Oviedo.

Telephone 985108720

Email: martaealvarez@gmail.com 


\begin{abstract}
Purpose: Among the methods used to diagnose COVID-19, those based on genomic detection by $\mathrm{q}(\mathrm{RT})$-PCR are the most sensitive. To perform these assays, a previous genome extraction of the sample is required. The dramatic increase in the number of SARS-CoV-2 detection assays has increased the demand for extraction reagents hindering the supply of commercial reagents. Homemade reagents and procedures could be an alternative.

Methods: Nasopharyngeal samples were extracted by seven different methods as well as the automatic method MagNaPure96, to detect SARS-CoV-2.

Results: All protocols show sensitivity higher than $87 \%$, in comparison with reference method, for detecting SARS-CoV-2 as well as human $\beta$ - globin.

Conclusions: Our results support that these procedures, using common and cheap reagents, are effective to extract RNA (from SARS-CoV-2) or DNA (from human $\beta$ globin) genome from nasopharyngeal swabs. Furthermore, these procedures could be easily adopted by routine diagnostic laboratories to implement detection methods to help to fight against COVID-19 pandemic.
\end{abstract}

\title{
Keywords
}

SARS-CoV-2; COVID-19; genome extraction; In-house protocol; Heat extraction. 
2 In December 2019, Chinese health authorities identified the new betacoronavirus SARS-

3 CoV-2 as the cause of the respiratory illness COVID-19, which was declared pandemic by

$4 \quad$ World Health Organization in January $2020(1,2)$. High sensitivity diagnostic methods to

5 detect and contain potential outbreaks are required to fight against this pandemic.

6 Among these methods, those based on genomic detection by quantitative

7 retrotranscriptase (RT)-PCR have been proved to be the best for a quick and sensitive

8 detection of COVID-19 infected patients. To perform these assays, a previous genome

9 extraction of the sample is required. This step is essential since both quantity and quality

10 of the genome obtained could affect the further amplification process (3). Because of

11 that, some studies comparing different commercial and/or manual procedures for

12 genome extraction from different type of samples, such as fecal, blood or respiratory

13 specimens have been reported (4-6).

14 The current global health emergency due to SARS-CoV-2 pandemic has caused a

15 dramatic increase in the number of detection assays performed by diagnostic

16 laboratories and, therefore, a huge demand for extraction reagents making difficult the

17 supply of commercial reagents. Homemade reagents and manual procedures are an

18 alternative. Our team has conducted a comparative study between seven manual

19 procedures with commercial and homemade reagents.

20 The aim was to evaluate alternative protocols to commercial genome extraction

21 procedures to be used for SARS-CoV-2 detection by routine diagnostic laboratories. 
Samples: A total of 58 nasopharyngeal samples from patients with suspicious of SARS-

CoV-2 infection were collected. The original volume of each sample $(200 \mu \mathrm{L})$ was diluted

8 times to a final volume of $1.6 \mathrm{~mL}$ to get enough volume to perform all extraction procedures.

Genome extraction procedures: The automatic extraction method MagNa Pure 96 System (Roche, Ginebra, Switzerland), which is usually developed in the laboratory, was taken as reference. Seven different procedures were carried out manually. MagNA Pure 96 System and MagNA Pure 32 System (Roche) were performed using commercial reagents following manufacturer's instructions. Other four protocols named as OneStep Method A (OSM-A), One-Step Method B (OSM-B), Two-Step Method (TSM) and protocol, which is based on heat treatment was performed in a SureCycler 8800 (Agilent Technologies, Santa Clara, CA) and no reagents were necessary. Protocols and reagents of the different genome extraction procedures were tested one time for each sample and are shown in Table 1.

qRT-PCR: all extracted samples were tested with a multiple qRT-PCR directed to two regions of the SARS-CoV 2 genome (Orf1ab and $N$ gene), as well as the human $\beta$ - globin gen. Briefly, $5 \mu \mathrm{l}$ of sample, previously extracted by any of the tested methods, were added to $10 \mu \mathrm{l}$ of TaqMan Fast 1-Step Master Mix (Life technologies, Carlsbad, CA) supplemented with a mixture of primers (Thermo Fisher Scientific, Walthman, MA) and taqman MGB probes (Applied Biosystems, Foster City, CA) (Table 2). Amplification and 
System (Applied Biosystems). The cycling protocol was as follows: (50ㄷ, 20 min; 95ㄷ $\mathrm{C}$, $5 \mathrm{~min} ; 45$ cycles of $95^{\circ} \mathrm{C}, 10 \mathrm{sec} ; 55^{\circ} \mathrm{C}, 15 \mathrm{sec}$ and $\left.60^{\circ} \mathrm{C}, 30 \mathrm{sec}\right)$.

Statistical studies: A T-Student test, whose null hypothesis was that in-house protocols works in the same way that the reference method, with a p-value of 0.05 , was performed.

\section{Results}

Nasopharyngeal samples were extracted by seven different manual methods, as well as by the automatic extraction method MagNA Pure 96 used as reference, and amplified by qRT-PCR. According to the reference automatic method, $\beta$-globin gene was detected in all samples, being 30 of them also positive to SARS-CoV-2 genome. The cycle threshold (Ct) of each amplification and mean, rank and $95 \% \mathrm{Cl}$ of each method are shown in tables 3 and 4.

The sensitivity for SARS-CoV-2 detection of the manual methods was calculated by comparison to automatic method. For OSM-A sensitivity was $93.3 \%$ while for MP32 achieved $100 \%$. The limits of $95 \% \mathrm{Cl}$ were 30.16 and 32.7 in automatic method MP96. By mean difference, in MP32 were 32.34 and 33.66 and in OSM-A were 33.36 and 34.84 . The sensitivity for $\beta$-globin detection of the manual methods was also calculated by comparison to automatic method. For "Bikop" extraction sensitivity was $87.9 \%$ while for OSM-A reached $100 \%$. The limits of $95 \% \mathrm{Cl}$ were 30.16 and 32.38 in automatic method MP96. By mean difference, in "Bikop" extraction were 34.53 and 36.25 and in MP32 were 35.96 and 37.58 . 


\section{Discussion}

The expansion of the outbreak of the new coronavirus SARS-CoV-2 and the global pandemic situation caused by its spread has provoked the need of a quick and efficient tool for diagnosis of viral disease. Genome amplification based on PCR has been raised as the best method and as important as the amplification process is the previous genome extraction step. In this process, the viral genome as well as cellular genome is purified. Both genomes are used to amplify and quantify the viral genome and the human gene $\beta$-globin, respectively. The ratio between the two values allows calculating a normalized viral load and determining the sample quality $(8,9)$.

Considering that the use of PCR commercial kits is very usual in most clinical laboratories, the supply of reagents during pandemic peak was a common problem. Trying to obtain a solution for future similar situations, several extraction genome procedures were tested with the idea of obtaining a method to be included in manual or even automatic diagnostic procedures used routinely in clinical laboratories. Similar methods were also developed by other authors (10-12). In our study, the sensitivity for SARS-CoV-2 of the tested procedures was higher than $93 \%$. These results were also observed in $\beta$-globin gene amplification, were all methods bowed a sensitivity higher than $87 \%$. According to these data, any method has enough sensitivity and can be used routinely in a clinical laboratory.

Data were a little bit worse when Cts of SARS-CoV-2 were compared with reference methods, Also, higher Cts were observed in $\beta$-globin detection. Because sensitivity was decreased in both targets (SARS-CoV-2 and $\beta$-globin), viral load was minimally underestimated, no more than 0.5 log considering that each 3 Cts means a difference in the viral load of $1 \mathrm{log}$. A possible explanation to these results is that manual processing 
92 is less precise and more prone to error than automatic methods using a robot.

93 Automatization of these procedures can solve this situation.

94 One limitation of the tested procedures can be that they are not quick enough. A possible alternative is cut time at least in half for washing steps. In preliminary studies in our laboratory changes in results were not observed. The main advantage is the use of common reagents making possible these procedures can be easily adapted by any other laboratory directly or changing the reagents for other with similar properties. Furthermore, these procedures could also be used for the extraction of RNA or DNA genome of other viruses.

Heat extraction method, whose sensitivities for SARS-CoV-2 and $\beta$-globin were $96.7 \%$ and $87.9 \%$, respectively, can be used for a quick screening of infected patients with suspicious of a high viral load. Recent reports using a similar method support the viability of this technology to SARS-CoV-2 genome extraction $(13,14)$. Considering that this is the quickest (only 15 minutes), cheapest and easiest (only need a thermoblock) protocol of all the tested procedures, it appears as a clear alternative to be implemented in small diagnostic laboratories, favoring a decentralization of SARS-CoV-2 diagnostic and desaturating central hospitals. In this method, Cts were similar than in others, and good enough for using when samples level is high.

In summary, in-house procedures evaluated can substitute commercial techniques performing a successful SARS-CoV-2 genome extraction using common and cheap reagents. On the other hand, human genome can also be successfully extracted allowing the use of $\beta$-globin gene as sample quality control and for normalized viral load calculus. These procedures could be easily adopted by clinical laboratories and be used to extract human samples with suspect of any viral presence. It is worth to note that Heat 
117 diagnosis laboratories.

120 Acknowledgments

121 To ASCOL (Asturiana de Control Lechero) for partially financing this project, supporting 122 research as the key to overcoming the pandemic.

123

124 Declarations

125 Funding: this research was partially granted by ASCOL (Asturiana de Control Lechero).

126 Conflict of interest statement: the authors declare no potential conflicts of interest.

127 Availability: of data and material: Not applicable

128 Code Availability: Not applicable 


\section{References}

1. Wang C, Horby PW, Hayden FG, Gao GF. A novel coronavirus outbreak of global health concern [published correction appears in Lancet. 2020 Jan 29]. Lancet. 2020;395(10223):470-473. doi:10.1016/S0140-6736(20)30185-9

2. WHO web page https://www.who.int/es/news-room/detail/27-04-2020-whotimeline---covid-19 $(06 / 12 / 2020)$

3. Rodríguez A, Duyvejonck H, Van Belleghem JD, et al. Comparison of procedures for RNA-extraction from peripheral blood mononuclear cells. PLoS One. 2020;15(2):e0229423. Published 2020 Feb 21. doi:10.1371/journal.pone.0229423

4. Verheyen J, Kaiser R, Bozic M, Timmen-Wego M, Maier BK, Kessler HH. Extraction of viral nucleic acids: comparison of five automated nucleic acid extraction platforms. $J$ Clin Virol. 2012;54(3):255-259. doi:10.1016/j.jcv.2012.03.008

5. Mengelle C, Mansuy JM, Da Silva I, Davrinche C, Izopet J. Comparison of 2 highly automated nucleic acid extraction systems for quantitation of human cytomegalovirus in whole blood. Diagn Microbiol Infect Dis. 2011;69(2):161-166. doi:10.1016/j.diagmicrobio.2010.08.011

6. Yang G, Erdman DE, Kodani M, Kools J, Bowen MD, Fields BS. Comparison of commercial systems for extraction of nucleic acids from DNA/RNA respiratory pathogens.J Virol Methods. 2011;171(1):195-199. doi:10.1016/j.jviromet.2010.10.024

7. CDC https://www.cdc.gov/coronavirus/2019-ncov/lab/rt-pcr-panel-primerprobes.html

8. Lescure FX, Bouadma L, Nguyen D, et al. Clinical and virological data of the first cases of COVID-19 in Europe: a case series [published correction appears in Lancet Infect 
Dis. 2020 May 19;:] [published correction appears in Lancet Infect Dis. 2020 Jun;20(6):e116]. Lancet Infect Dis. 2020;20(6):697-706. doi:10.1016/S14733099(20)30200-0

9. Gómez-Novo M, Boga JA, Álvarez-Argüelles ME, et al. Human respiratory syncytial virus load normalized by cell quantification as predictor of acute respiratory tract infection. J Med Virol. 2018;90(5):861-866. doi:10.1002/jmv.25020

10. Yamada O, Matsumoto $T$, Nakashima M, et al. A new method for extracting DNA or RNA for polymerase chain reaction.J Virol Methods. 1990;27(2):203-209. doi:10.1016/0166-0934(90)90136-4

11. Arruda E, Hayden FG. Detection of human rhinovirus RNA in nasal washings by PCR. Mol Cell Probes. 1993;7(5):373-379. doi:10.1006/mcpr.1993.1055

12. He H, Li R, Chen $\mathrm{Y}$, et al. Integrated DNA and RNA extraction using magnetic beads from viral pathogens causing acute respiratory infections. Sci Rep. 2017;7:45199. Published 2017 Mar 23. doi:10.1038/srep45199

13. Merindol N, Pépin G, Marchand C, et al. SARS-CoV-2 detection by direct rRT-PCR without RNA extraction.J Clin Virol. 2020;128:104423. doi:10.1016/j.jcv.2020.104423

14. Mancini F, Barbanti F, Scaturro M, et al. Laboratory management for SARS-CoV-2 detection: a user-friendly combination of the heat treatment approach and rt-Realtime PCR testing. Emerg Microbes Infect. 2020;9(1):1393-1396. doi:10.1080/22221751.2020.1775500 
Table 1: Extraction procedures steps

\begin{tabular}{|c|c|c|c|c|c|c|c|}
\hline Steps & $\begin{array}{l}\text { MagNa Pure } 96^{1} \\
\text { (MP96) }\end{array}$ & $\begin{array}{l}\text { MagNa Pure } 32^{1} \\
\text { (MP32) }\end{array}$ & $\begin{array}{c}\text { One-step } \\
\text { method A } \\
(\text { OSM-A) } \\
\end{array}$ & $\begin{array}{c}\text { One-step } \\
\text { method B } \\
\text { (OSM-B) } \\
\end{array}$ & $\begin{array}{l}\text { Two-steps } \\
\text { method } \\
\text { (TSM) }\end{array}$ & $\begin{array}{l}\text { "Bikop" } \\
\text { method }\end{array}$ & $\begin{array}{c}\text { Heat } \\
\text { Extraction }\end{array}$ \\
\hline 1 & $\begin{array}{l}\text { Lysis buffer MP96 } \\
\text { Incubate }\left(10^{\prime}-\mathrm{RT}^{*}\right)\end{array}$ & $\begin{array}{l}\text { Lysis buffer MP32 } \\
\text { Incubate }\left(10^{\prime}-\mathrm{RT}\right)\end{array}$ & $\begin{array}{c}\text { Lysis buffer } 1^{2} \\
\text { Incubate }\left(10^{\prime}-\mathrm{RT}\right)\end{array}$ & $\begin{array}{c}\text { Lysis buffer } 1^{3} \\
\text { Incubate }\left(10^{\prime}-\mathrm{RT}\right)\end{array}$ & $\begin{array}{c}\text { Lysis buffer } 1^{4} \\
\text { Incubate }\left(10^{\prime}-\mathrm{RT}\right)\end{array}$ & $\begin{array}{c}\text { Lysis buffer } 1^{2} \\
\text { Incubate }\left(10^{\prime}-\mathrm{RT}\right)\end{array}$ & Hot Spot $\left(10^{\prime}-95^{\circ} \mathrm{C}\right)$ \\
\hline 2 & & & & & $\begin{array}{c}\text { Lysis Buffer } 2^{2} \\
\text { Incubate }\left(10^{\prime}-\mathrm{RT}\right)\end{array}$ & & Freeze $\left(5^{\prime}-4^{\circ} \mathrm{C}\right)$ \\
\hline 3 & $\begin{array}{c}\text { Binding Buffer MP96 } \\
\text { Incubate in shaker } \\
\left(10^{\prime}-\mathrm{RT}\right) \\
\text { Magnetize* \& }^{*} \\
\text { Remove SN* }\end{array}$ & $\begin{array}{c}\text { Binding Buffer MP32 } \\
\text { Incubate in shaker } \\
\left(10^{\prime}-\text { RT }\right) \\
\text { Magnetize \& } \\
\text { Remove SN }\end{array}$ & $\begin{array}{c}\text { Binding Buffer } \\
\text { Incubate in shaker } \\
\left(10^{\prime}-\text { RT }\right) \\
\text { Magnetize \& } \\
\text { Remove SN }\end{array}$ & $\begin{array}{c}\text { Binding Buffer } \\
\text { Incubate in shaker } \\
\left(10^{\prime}-\mathrm{RT}\right) \\
\text { Magnetize \& } \\
\text { Remove SN }\end{array}$ & $\begin{array}{c}\text { Binding Buffer }^{5} \\
\text { Incubate in shaker } \\
\left(10^{\prime}-\mathrm{RT}\right) \\
\text { Magnetize \& } \\
\text { Remove SN }\end{array}$ & $\begin{array}{c}\text { Binding Buffer } \\
\text { Incubate in shaker } \\
\left(10^{\prime}-\text { RT) }\right. \\
\text { Magnetize \& } \\
\text { Remove SN } \\
\end{array}$ & \\
\hline 4 & $\begin{array}{c}\text { Wash Buffer 1 MP96 } \\
\text { Incubate (10' - RT) } \\
\text { Magnetize \& } \\
\text { Remove SN }\end{array}$ & $\begin{array}{c}\text { Wash Buffer 1 MP32 } \\
\text { Incubate }\left(10^{\prime}-\text { RT) }\right. \\
\text { Magnetize \& } \\
\text { Remove SN }\end{array}$ & $\begin{array}{c}\text { Wash Buffer } 1^{6} \\
\text { Incubate }\left(10^{\prime}-\mathrm{RT}\right) \\
\text { Magnetize \& } \\
\text { Remove SN }\end{array}$ & $\begin{array}{c}\text { Wash Buffer } 1^{6} \\
\text { Incubate }\left(10^{\prime}-\mathrm{RT}\right) \\
\text { Magnetize \& } \\
\text { Remove SN }\end{array}$ & $\begin{array}{c}\text { Wash Buffer } 1^{6} \\
\text { Incubate }\left(10^{\prime}-\mathrm{RT}\right) \\
\text { Magnetize \& } \\
\text { Remove SN }\end{array}$ & $\begin{array}{c}\text { Wash Buffer } 1^{6} \\
\text { Incubate }\left(10^{\prime}-\mathrm{RT}\right) \\
\text { Magnetize \& } \\
\text { Remove SN }\end{array}$ & \\
\hline 5 & $\begin{array}{c}\text { Wash Buffer } 2 \text { MP96 } \\
\text { Incubate }\left(10^{\prime}-\text { RT }\right) \\
\text { Magnetize \& } \\
\text { Remove SN } \\
\end{array}$ & $\begin{array}{c}\text { Wash Buffer } 2 \text { MP32 } \\
\text { Incubate }\left(10^{\prime}-\text { RT }\right) \\
\text { Magnetize \& } \\
\text { Remove SN } \\
\end{array}$ & $\begin{array}{c}\text { Wash Buffer } 2^{7} \\
\text { Incubate }\left(10^{\prime}-\mathrm{RT}\right) \\
\text { Magnetize \& } \\
\text { Remove SN }\end{array}$ & $\begin{array}{c}\text { Wash Buffer } 2^{7} \\
\text { Incubate }\left(10^{\prime}-\mathrm{RT}\right) \\
\text { Magnetize \& } \\
\text { Remove SN }\end{array}$ & $\begin{array}{c}\text { Wash Buffer } 2^{7} \\
\text { Incubate }\left(10^{\prime}-\mathrm{RT}\right) \\
\text { Magnetize \& } \\
\text { Remove SN }\end{array}$ & $\begin{array}{c}\text { Wash Buffer } 2^{7} \\
\text { Incubate }\left(10^{\prime}-\mathrm{RT}\right) \\
\text { Magnetize \& } \\
\text { Remove SN }\end{array}$ & \\
\hline 6 & $\begin{array}{c}\text { Wash Buffer } 3 \text { MP96 } \\
\text { Incubate (10' - RT) } \\
\text { Magnetize \& } \\
\text { Remove SN } \\
\end{array}$ & $\begin{array}{c}\text { Wash Buffer } 3 \text { MP32 } \\
\text { Incubate }\left(10^{\prime}-\text { RT }\right) \\
\text { Magnetize \& } \\
\text { Remove SN } \\
\end{array}$ & $\begin{array}{c}\text { Wash Buffer } 3^{8} \\
\text { Incubate }\left(10^{\prime}-\mathrm{RT}\right) \\
\text { Magnetize \& } \\
\text { Remove SN } \\
\end{array}$ & $\begin{array}{c}\text { Wash Buffer } 3^{8} \\
\text { Incubate }\left(10^{\prime}-\mathrm{RT}\right) \\
\text { Magnetize \& } \\
\text { Remove SN } \\
\end{array}$ & $\begin{array}{c}\text { Wash Buffer } 3^{8} \\
\text { Incubate }\left(10^{\prime}-\mathrm{RT}\right) \\
\text { Magnetize \& } \\
\text { Remove SN } \\
\end{array}$ & & \\
\hline 7 & $\begin{array}{c}\text { Elution Buffer MP96 } \\
\text { Magnetize \& } \\
\text { Collect SN }\end{array}$ & $\begin{array}{c}\text { Elution Buffer MP32 } \\
\text { Magnetize \& } \\
\text { Collect SN }\end{array}$ & $\begin{array}{c}\text { Elution Buffer }^{9} \\
\text { Magnetize \& } \\
\text { Collect SN }\end{array}$ & $\begin{array}{c}\text { Elution Buffer }^{9} \\
\text { Magnetize \& } \\
\text { Collect SN }\end{array}$ & $\begin{array}{c}\text { Elution Buffer }^{9} \\
\text { Magnetize \& } \\
\text { Collect SN }\end{array}$ & $\begin{array}{c}\text { Elution Buffer }^{9} \\
\text { Magnetize \& } \\
\text { Collect SN }\end{array}$ & \\
\hline $\begin{array}{c}\text { Time } \\
\text { (estimate) }\end{array}$ & $500^{\prime}$ & $\mathbf{5 0}$ & $\mathbf{5 0}$ & $\mathbf{5 0}$ & $60^{\prime}$ & $40^{\prime}$ & $15^{\prime}$ \\
\hline
\end{tabular}

*RT: Room Temperature

*Using a 96R Ring Magnet Plate (Alpaqua, Beverly, MA)

*SN: supernatant

${ }^{1}$ Reagents and volumes suggested by manufacturer were used in these protocols.

${ }^{2}$ Lysis Buffer 1: $200 \mu \mathrm{L}$ GIT (GIT 4M + sarcosyl 0,5\% + sodium citrate $25 \mathrm{mM}$ ) and $10 \mu \mathrm{L}$ $\mathrm{pK}(10 \mu \mathrm{g} / \mu \mathrm{L})$

${ }^{3}$ Lysis Buffer 2: $200 \mu \mathrm{L}$ GIT, $10 \mu \mathrm{L}$ pK and $200 \mu \mathrm{L}$ SDS (SDS 0,5\% + Tris (pH 8) $10 \mathrm{mM}+$ EDTA $1 \mathrm{mM}$

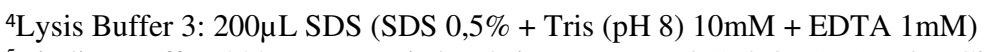
${ }^{5}$ Binding Buffer: $200 \mu \mathrm{L}$ magnetic beads in Isopropanol $(1,5 \mathrm{~g} / \mathrm{mL}$ ), (Roche, Ginebra, Switzerland)

${ }^{6}$ Wash Buffer 1: 200 $\mu \mathrm{L}$ isopropanol

${ }^{7}$ Wash Buffer 2. 200 $\mathrm{LL}$ EtOH $80 \%$

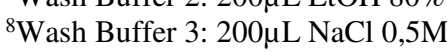

${ }^{9}$ Elution Buffer: $100 \mu \mathrm{L}$ water 
Table 2: Primers and taqman MGB probes used to detect SARS-CoV2 and human $\beta$-globin.

\begin{tabular}{|c|c|c|c|c|}
\hline Design & Gen & Function & Name & Sequence $\left(5^{\prime}-3^{\prime}\right)$ \\
\hline In-house & $\begin{array}{l}\text { ORF1ab } \\
\text { SARS-CoV2 }\end{array}$ & $\begin{array}{l}\text { Forward primer } \\
\text { Reverse primer } \\
\text { MGB FAM probe }\end{array}$ & $\begin{array}{l}\text { CoV-2-OVI-S } \\
\text { CoV-2-OVI-A } \\
\text { CoV-2-OVI-FAM }\end{array}$ & $\begin{array}{l}\text { ATCAAGTTAATGGTTACCCTAACATGT } \\
\text { AACCTAGCTGTAAAGGTAAATTGGTACC } \\
\text { CCGCGAAGAAGCTA }\end{array}$ \\
\hline $\mathrm{CDC}^{1}$ & $\begin{array}{l}\mathrm{N} \\
\text { SARS-CoV2 }\end{array}$ & $\begin{array}{l}\text { Forward primer } \\
\text { Reverse primer } \\
\text { MGB VIC probe }\end{array}$ & $\begin{array}{l}\text { 2019-nCoV-N1-F } \\
\text { 2019-nCoV-N1-R } \\
\text { 2019-nCoV-N1-P-VIC } \\
\end{array}$ & $\begin{array}{l}\text { GACCCCAAAATCAGCGAAAT } \\
\text { TCTGGTTACTGCCAGTTGAATCTG } \\
\text { CCGCATTACGTTTGGT }^{2}\end{array}$ \\
\hline In-house & $\begin{array}{l}\beta \text {-globin } \\
\text { Human }\end{array}$ & $\begin{array}{l}\text { Forward primer } \\
\text { Reverse primer } \\
\text { MGB Cy } 5 \text { probe }\end{array}$ & $\begin{array}{l}\text { Beta-TR-S } \\
\text { Beta-TR-A } \\
\text { Beta-Cy5 }\end{array}$ & $\begin{array}{l}\text { ACACAACTGTGTTCACTAGC } \\
\text { CCAACTTCATCCACGTTCACC } \\
\text { TGCATCTGACTCCTGAGGA }\end{array}$ \\
\hline
\end{tabular}

${ }^{1}$ Sequences published by Center for Disease Control and Prevention (CDC) (7) 
Table 3:Results of amplification of SARS-CoV-2 and human B-globin (Ct SARS-CoV-2/Ct B-globin)

\begin{tabular}{|c|c|c|c|c|c|c|c|c|}
\hline Samples & $\begin{array}{c}\text { MP96 } \\
\text { (automated) }\end{array}$ & $\begin{array}{c}\text { MP96 } \\
\text { (handmade) }\end{array}$ & MP32 & OSM-A & OSM-B & TSM & $\begin{array}{l}\text { "Bikop" } \\
\text { method }\end{array}$ & $\begin{array}{c}\text { Heat } \\
\text { Extraction }\end{array}$ \\
\hline 10 & $24 / 36$ & $30 / 40$ & $30 / 40$ & $31 / 40$ & $30 / 40$ & $29 / 40$ & $29 / 36$ & $31 / 38$ \\
\hline 20 & $24 / 29$ & $30 / 33$ & $30 / 36$ & 35 / 34 & 31 / 34 & $29 / 36$ & $27 / 32$ & 30 / 34 \\
\hline 3음 & 25 / 34 & $29 / 38$ & $31 / 40$ & 31 / 39 & $31 / 40$ & 32 / 39 & $27 / 35$ & $27 / 38$ \\
\hline $4 \stackrel{0}{\circ}$ & $26 / 30$ & 31 / 34 & 31 / 37 & 31 / 39 & 33 / 36 & 30 / 34 & 30 / 35 & $32 / 36$ \\
\hline 5음 & 27 / 31 & 31 / 35 & 30 / 37 & 32 / 40 & 32 / 35 & $31 / 35$ & 34 / 36 & 33 / 40 \\
\hline 6음 & $27 / 29$ & 33 / 34 & 32 / 37 & 33 / 36 & 34 / 36 & $31 / 35$ & 31 / 34 & $31 / 36$ \\
\hline 70 & $28 / 39$ & $33 / 40$ & $30 / 40$ & 32 / 40 & 32 / 40 & $31 / 40$ & 32 / 40 & 30 / 40 \\
\hline 80 & 30 / 29 & 34 / 31 & 33 / 37 & $36 / 35$ & 32 / 34 & 33 / 35 & 31 / 32 & 34 / 33 \\
\hline 9응 & 30 / 40 & 31 / 36 & $32 / 40$ & $31 / 40$ & 31 / 40 & 30 / 40 & 31 / 40 & 32 / 40 \\
\hline $10 ㅇ$ & 31 / 31 & 33 / 38 & 33 / 39 & 33 / 34 & 34 / 34 & 34 / 36 & 34 / 34 & 34 / 33 \\
\hline 110 & 32 / 29 & 33 / 32 & 34 / 35 & 34 / 34 & 33 / 36 & $33 / 33$ & 33 / 33 & $33 / 40$ \\
\hline $12 \circ$ & 32 / 34 & 37 / 39 & 34 / 37 & 36 / 39 & $35 / 38$ & 39 / 37 & $37 / 35$ & $36 / 35$ \\
\hline $13 ㅇ$ & 32 / 33 & 34 / 38 & 31 / 34 & $33 / 40$ & 32 / 40 & 32 / 38 & 33 / 39 & 34 / 40 \\
\hline $14 \stackrel{0}{0}$ & 32 / 36 & $31 / 40$ & 32 / 38 & 33 / 40 & 32 / 40 & 32 / 38 & 32 / 40 & 34 / 40 \\
\hline 150 & 33 / 37 & $33 / 40$ & 34 / 37 & 39 / 40 & $38 / 40$ & $33 / 40$ & $33 / 38$ & $34 / 40$ \\
\hline 160 & 33 / 37 & $34 / 40$ & $33 / 40$ & $36 / 40$ & $36 / 40$ & $0 / 40$ & $33 / 40$ & 34 / 40 \\
\hline 170 & 33 / 27 & $34 / 40$ & $33 / 32$ & 34 / 35 & 35 / 32 & $33 / 35$ & 33 / 31 & $0 / 34$ \\
\hline $18^{\circ}$ & 33 / 35 & $36 / 38$ & $35 / 40$ & $35 / 40$ & $35 / 40$ & $36 / 40$ & $0 / 40$ & $37 / 40$ \\
\hline 190 & $0(31) * / 33$ & $33 / 40$ & $34 / 40$ & $0 / 38$ & 33 / 37 & $34 / 36$ & $33 / 36$ & $34 / 35$ \\
\hline 200 & 33 / 36 & $34 / 40$ & $34 / 40$ & 34 / 37 & $33 / 40$ & $33 / 40$ & $33 / 40$ & $34 / 40$ \\
\hline 210 & 34 / 34 & 34 / 36 & $36 / 40$ & 37 / 39 & $36 / 40$ & $34 / 38$ & $38 / 38$ & $36 / 40$ \\
\hline $22 \circ$ & 34 / 32 & $33 / 35$ & $33 / 40$ & $0 / 35$ & 35 / 37 & $33 / 36$ & $35 / 35$ & 35 / 34 \\
\hline $23 ㅇ$ & $34 / 23$ & $34 / 40$ & 34 / 39 & $34 / 40$ & $34 / 40$ & $33 / 37$ & $33 / 40$ & $34 / 40$ \\
\hline $24 ㅇ$ & $34 / 28$ & 37 / 31 & $37 / 33$ & 34 / 32 & 34 / 32 & 34 / 31 & $34 / 30$ & $35 / 28$ \\
\hline 250 & 34 / 40 & 34 / 40 & 34 / 40 & 35 / 40 & $36 / 40$ & $34 / 40$ & $35 / 40$ & $35 / 40$ \\
\hline 260 & $35 / 40$ & $36 / 40$ & $35 / 40$ & $37 / 40$ & $36 / 39$ & $35 / 40$ & $39 / 40$ & $38 / 40$ \\
\hline $27 \stackrel{0}{0}$ & 35 / 35 & 35 / 40 & $36 / 40$ & $37 / 40$ & $37 / 40$ & $37 / 40$ & 37 / 39 & $36 / 40$ \\
\hline 280 & $36 / 38$ & $34 / 40$ & $33 / 40$ & $34 / 40$ & 35 / 40 & $35 / 40$ & 35 / 40 & 35 / 40 \\
\hline 290 & $0(35)^{*} / 27$ & 34 / 30 & 33 / 31 & 35 / 31 & 35 / 32 & $33 / 30$ & $0 / 32$ & 38 / 31 \\
\hline $30 \circ$ & $0(36) * / 33$ & $0 / 0$ & 33 / 39 & 33 / 39 & $0 / 40$ & $35 / 38$ & $34 / 40$ & $35 / 38$ \\
\hline 310 & $-/ 22$ & $-/ 26$ & $-/ 29$ & $-/ 28$ & $-/ 28$ & $-/ 28$ & $-/ 26$ & $-/ 25$ \\
\hline $32 \circ$ & $-/ 23$ & $-/ 0$ & $-/ 35$ & $-/ 33$ & $-/ 31$ & $-/ 33$ & $-/ 32$ & $-/ 33$ \\
\hline $33 ㅇ$ & $-/ 24$ & $-/ 32$ & $-/ 36$ & $-/ 33$ & $-/ 33$ & $-/ 32$ & $-/ 34$ & $-/ 33$ \\
\hline $34 ㅇ$ & $-/ 25$ & $-/ 33$ & $-/ 37$ & $-/ 34$ & $-/ 34$ & $-/ 34$ & $-/ 35$ & $-/ 33$ \\
\hline 35 응 & $-/ 26$ & $-/ 31$ & $-/ 32$ & $-/ 30$ & $-/ 31$ & $-/ 30$ & $-/ 30$ & $-/ 31$ \\
\hline 360 & $-/ 27$ & $-/ 32$ & $-/ 35$ & $-/ 31$ & $-/ 32$ & $-/ 32$ & $-/ 34$ & $-/ 32$ \\
\hline $37 ㅇ$ & $-/ 27$ & $-/ 40$ & $-/ 31$ & $-/ 31$ & $-/ 30$ & $-/ 30$ & $-/ 30$ & $-/ 38$ \\
\hline 380 & $-/ 28$ & $-/ 32$ & $-/ 40$ & $-/ 33$ & $-/ 34$ & $-/ 33$ & $-/ 35$ & $-/ 34$ \\
\hline 390 & $-/ 28$ & $-/ 0$ & $-/ 40$ & $-/ 35$ & $-/ 36$ & $-/ 32$ & $-/ 33$ & $-/ 33$ \\
\hline 40 & $-/ 28$ & $-/ 0$ & $-/ 36$ & $-/ 32$ & $-/ 33$ & $-/ 32$ & $-/ 35$ & $-/ 33$ \\
\hline $41 ㅇ$ & $-/ 29$ & $-/ 32$ & $-/ 32$ & $-/ 34$ & $-/ 35$ & $-/ 0$ & $-/ 0$ & $-/ 0$ \\
\hline $42 \circ$ & $-/ 29$ & $-/ 0$ & $-/ 40$ & $-/ 33$ & $-/ 33$ & $-/ 35$ & $-/ 34$ & $-/ 37$ \\
\hline $43 ㅇ$ & $-/ 29$ & $-/ 31$ & $-/ 31$ & $-/ 32$ & $-/ 33$ & $-/ 33$ & $-/ 0$ & $-/ 0$ \\
\hline 440 & $-/ 29$ & $-/ 33$ & $-/ 34$ & $-/ 33$ & $-/ 33$ & $-/ 34$ & $-/ 33$ & $-/ 32$ \\
\hline 450 & $-/ 30$ & $-/ 33$ & $-/ 33$ & $-/ 35$ & $-/ 37$ & $-/ 33$ & $-/ 0$ & $-/ 33$ \\
\hline 460 & $-/ 30$ & $-/ 36$ & $-/ 38$ & $-/ 34$ & $-/ 33$ & $-/ 38$ & $-/ 35$ & $-/ 0$ \\
\hline $47 ㅇ$ & $-/ 30$ & $-/ 34$ & $-/ 34$ & $-/ 33$ & $-/ 32$ & $-/ 33$ & $-/ 34$ & $-/ 31$ \\
\hline 480 & $-/ 31$ & $-/ 35$ & $-/ 35$ & $-/ 39$ & $-/ 37$ & $-/ 35$ & $-/ 0$ & $-/ 32$ \\
\hline 490 & $-/ 31$ & $-/ 34$ & $-/ 40$ & $-/ 34$ & $-/ 34$ & $-/ 37$ & $-/ 35$ & $-/ 40$ \\
\hline $50 ㅇ$ & $-/ 31$ & $-/ 33$ & $-/ 33$ & $-/ 36$ & $-/ 36$ & $-/ 35$ & $-/ 0$ & $-/ 0$ \\
\hline 51 & $-/ 31$ & $-/ 33$ & $-/ 39$ & $-/ 36$ & $-/ 34$ & $-/ 36$ & $-/ 0$ & $-/ 34$ \\
\hline 52 & $-/ 32$ & $-/ 37$ & $-/ 37$ & $-/ 33$ & $-/ 34$ & $-/ 35$ & $-/ 33$ & $-/ 32$ \\
\hline 53으 & $-/ 32$ & $-/ 33$ & $-/ 33$ & $-/ 35$ & $-/ 35$ & $-/ 31$ & $-/ 35$ & $-/ 36$ \\
\hline $54 ㅇ$ & $-/ 32$ & $-/ 35$ & $-/ 35$ & $-/ 40$ & $-/ 0$ & $-/ 35$ & $-/ 35$ & $-/ 38$ \\
\hline 55으 & $-/ 33$ & $-/ 36$ & $-/ 40$ & $-/ 37$ & $-/ 35$ & $-/ 36$ & $-/ 36$ & $-/ 0$ \\
\hline 560 & $-/ 34$ & $-/ 38$ & $-/ 38$ & $-/ 37$ & $-/ 0$ & $-/ 0$ & $-/ 0$ & $-/ 37$ \\
\hline $57 ㅇ$ & $-/ 34$ & $-/ 0$ & $-/ 0$ & $-/ 39$ & $-/ 37$ & $-/ 36$ & $-/ 36$ & $-/ 40$ \\
\hline 58 & $-/ 34$ & $-/ 0$ & $-/ 35$ & $-/ 39$ & $-/ 37$ & $-/ 33$ & $-/ 35$ & $-/ 0$ \\
\hline
\end{tabular}

*these samples were negative on the MP96 automated extraction, so the Ct before dilution (in parenthesis) was used 


\begin{tabular}{|c|c|c|c|c|c|c|c|c|}
\hline SARS CoV2 samples & $\begin{array}{c}\text { MP96 } \\
\text { (automated) }\end{array}$ & $\begin{array}{c}\text { MP96 } \\
\text { (manual) }\end{array}$ & MP32 & OSM-A & OSM-B & TSM & "Bikop"method & Heat Extraction \\
\hline Positives (sensitivity) & $30(100 \%)$ & $29(96.7 \%)$ & $30(100 \%)$ & $28(93.3 \%)$ & 29 (96.7\%) & $29(96.7 \%)$ & $28(93.3 \%)$ & $29(96.7 \%)$ \\
\hline Mean $\pm \sigma$ & $31.43 \pm 3.54$ & $33.27 \pm 2$ & $33 \pm 1.86$ & $34.1 \pm 2.08$ & $33.79 \pm 2.01$ & $33.03 \pm 2.26$ & $33.07 \pm 2.85$ & $33.82 \pm 2.44$ \\
\hline Range & {$[24-36]$} & {$[29-37]$} & {$[30-37]$} & [31 - 39] & {$[30-38]$} & [29-39] & {$[27-39]$} & {$[27-38]$} \\
\hline $95 \%$ IC & [30.16- 32.7] & [32.55- 33.99] & {$[32.34-33.66]$} & {$[33.36-34.84]$} & {$[33.07-34.51]$} & {$[32.22-33.84]$} & {$[32.05-34.09]$} & [32.95-34.69] \\
\hline Mean difference ${ }^{1}$ & - & 1.84 & 1.57 & 2.67 & 2.36 & 1.59 & 1.64 & 2.39 \\
\hline p-value & - & 0.03 & $<0.001$ & 0.04 & 0.01 & 0.06 & 0.27 & 0.01 \\
\hline$\beta$-globin samples & $\begin{array}{c}\text { MP96 } \\
\text { (automated) }\end{array}$ & $\begin{array}{c}\text { MP96 } \\
\text { (manual) }\end{array}$ & MP32 & OSM-A & OSM-B & TSM & "Bikop" method & Heat Extraction \\
\hline Positives (sensitivity) & $58(100 \%)$ & $51(87.9 \%)$ & $57(98.3 \%)$ & $58(100 \%)$ & $56(96.6 \%)$ & $56(96,6 \%)$ & $51(87.9 \%)$ & $51(87.9 \%)$ \\
\hline Mean $\pm \sigma$ & $31.27 \pm 4.31$ & $35.62 \pm 3.59$ & $36.77 \pm 3.14$ & $36.12 \pm 3.35$ & $35.87 \pm 3.29$ & $35.5 \pm 3.20$ & $35.39 \pm 3.32$ & $35.88 \pm 3.77$ \\
\hline Range & {$[22-40]$} & {$[26-40]$} & {$[29-40]$} & {$[28-40]$} & {$[28-40]$} & {$[28-40]$} & {$[26-40]$} & {$[25-40]$} \\
\hline $95 \%$ IC & {$[30.16-32.38]$} & {$[34.70-36.54]$} & {$[35.96-37.58]$} & {$[35.26-36.98]$} & {$[35.02-36.72]$} & {$[34.68-36.32]$} & {$[34.53-36.25]$} & {$[34.91-36.85]$} \\
\hline Mean difference ${ }^{1}$ & - & 4.35 & 5.5 & 4.84 & 4.6 & 4.23 & 4.12 & 4.61 \\
\hline p-value & - & 0.006 & $<0.001$ & $<0.001$ & $<0.001$ & $<0.001$ & 0.01 & $<0.001$ \\
\hline
\end{tabular}

${ }^{1}$ Difference between means of the manual and automatic methods. 